\title{
Cerebral Microbleeds in Autosomal Dominant Polycystic Kidney Disease
}

\author{
Li-Kai Tsai, ${ }^{\text {a }}$ Pei-Lung Chen, ${ }^{\mathrm{b}, \mathrm{c}}$ Hsin-Hsi Tsai, ${ }^{\mathrm{a}, \mathrm{d}}$ Ya-Fang Chen, ${ }^{\mathrm{e}, \mathrm{f}}$ Ping-Chun Wu, ${ }^{\mathrm{g}}$ Jiann-Shing Jeng, \\ Jenq-Wen Huang, ${ }^{\text {h }}$ Tzong-Shinn Chu, ${ }^{\text {h }}$ Juliana Tze-Wah Kao ${ }^{\text {h,i }}$ \\ aDepartment of Neurology, National Taiwan University Hospital, National Taiwan University College of Medicine, Taipei, Taiwan \\ bDepartment of Medical Genetics, National Taiwan University Hospital, National Taiwan University College of Medicine, Taipei, Taiwan \\ 'Graduate Institute of Medical Genomics and Proteomics, National Taiwan University College of Medicine, Taipei, Taiwan \\ dDepartment of Neurology, National Taiwan University Hospital Bei-Hu Branch, Taipei, Taiwan \\ 'Department of Medical Imaging, National Taiwan University Hospital, National Taiwan University College of Medicine, Taipei, Taiwan \\ fDepartment of Medical Imaging, National Taiwan University Hospital Hsin-Chu Branch, Hsin-Chu, Taiwan \\ ${ }^{9}$ Graduate Institute of Molecular Medicine, National Taiwan University College of Medicine, Taipei, Taiwan \\ hDepartment of Internal Medicine, National Taiwan University Hospital, National Taiwan University College of Medicine, Taipei, Taiwan \\ 'Department of Internal Medicine, FuJen Catholic University Hospital, Fu Jen Catholic University, New Taipei City, Taiwan
}

\section{Dear Sir:}

Autosomal dominant polycystic kidney disease (PKD) is characterized by multiple enlarged renal cysts that subsequently lead to renal insufficiency. ${ }^{1}$ While PKD is associated with intracranial aneurysm, ${ }^{2}$ which reflects large artery anomalies, it remains unknown whether there is also abnormality in cerebral small vessels. The aim of this study was to investigate the prevalence and determinants of cerebral microbleeds (CMBs) in a cohort of patients with PKD.

Fifty-five consecutive PKD patients without past history of stroke, and 45 control subjects who visited the neurology outpatient clinics with no prior stroke or family history of PKD, were prospectively enrolled for brain magnetic resonance imaging (MRI), including susceptibility-weighted imaging (SWI). Twenty-two PKD patients (40\%) received another follow-up brain MRI study. CMBs were defined as small, round or ovoid hypointensities with a diameter less than $1 \mathrm{~cm}$ on SWI. ${ }^{3} \mathrm{~A}$ subgroup of patients with PKD underwent genetic testing using next-generation sequencing to screen the entire PKD1 and PKD2 genes. Fisher's exact test and Student's t-test or Mann-Whitney $\mathrm{U}$ test were used to determine the differences between groups for categorical data and continuous data, respectively. The determinants for the presence of CMBs were analyzed using a logistic regression model. Two-tailed $P$-values of less than 0.05 were considered statistically significant (STATA software version 8.2, StataCorp., College Station, TX, USA). Detailed methods are available in Supplementary methods. ${ }^{1,3-12}$

Comparison of the 55 PKD patients with the 45 control subjects (Supplementary Table 1) showed that PKD patients had a lower estimated glomerular filtration rate (eGFR) level than control subjects. More patients in the PKD group had CMB (27.3\% vs. $6.7 \%, P=0.009)$, intracranial aneurysm (10.9\% vs. $0 \%, P=0.031$ ) and hypertension than in the control group. Among 15 PKD patients with CMBs (mean age, $58.4 \pm 10.8$ years; range, 30 to 73 ), seven patients (46.7\%) had CMBs located exclusively at the lobar regions, five (33.3\%) had CMBs located exclusively at the deep regions and three (20\%) had lobar and deep CMBs (mixed location). The number of CMBs for each patient ranged from 1 to 6 , with a total count of $40 \mathrm{CMBs}$ for all patients; 22 (55\%) of the CMBs were in the lobar regions (12 in the frontal, five in temporal, four in occipital, and one in parietal regions) and 18 (45\%) were in the deep regions (eight in the cerebellum, five in basal ganglion, three in brainstem, and two in thalamus) (Figure 1).

Comparing the characteristics of PKD patients with and without CMBs (Supplementary Table 2), PKD patients with CMBs were older than those without $(58.4 \pm 10.8$ years vs. $48.7 \pm 9.8$ years, $P=0.002$ ). In univariable analysis of 100 patients and controls, age and the diagnosis of PKD were the positive determi- 
Abdominal CT
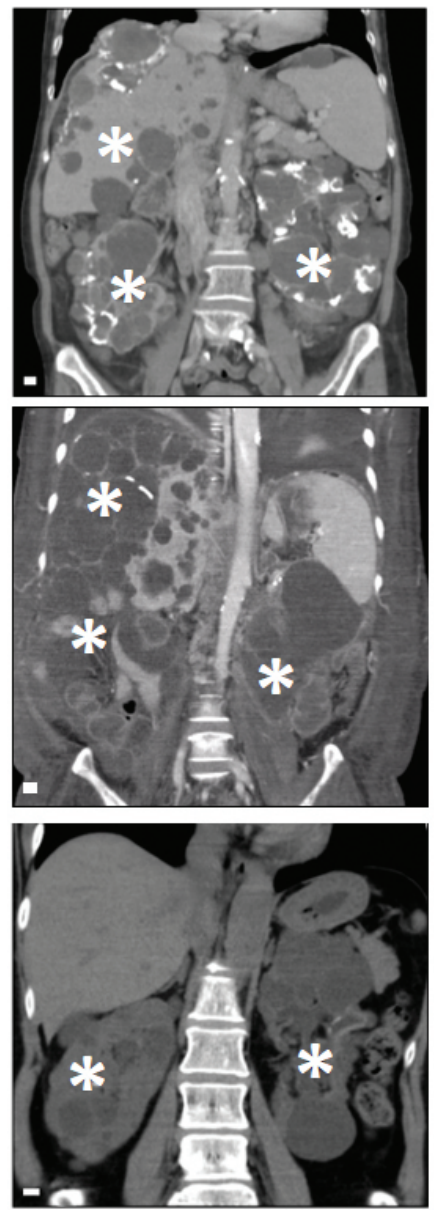

SWI
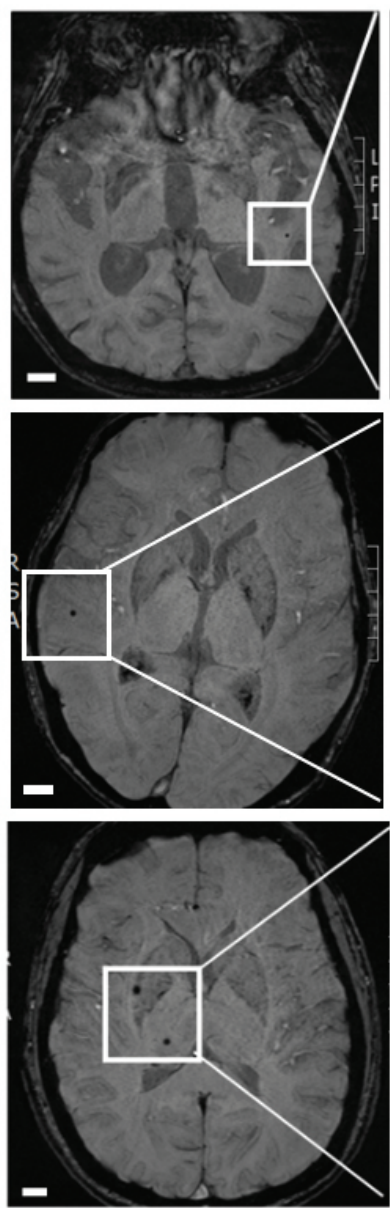

TOF
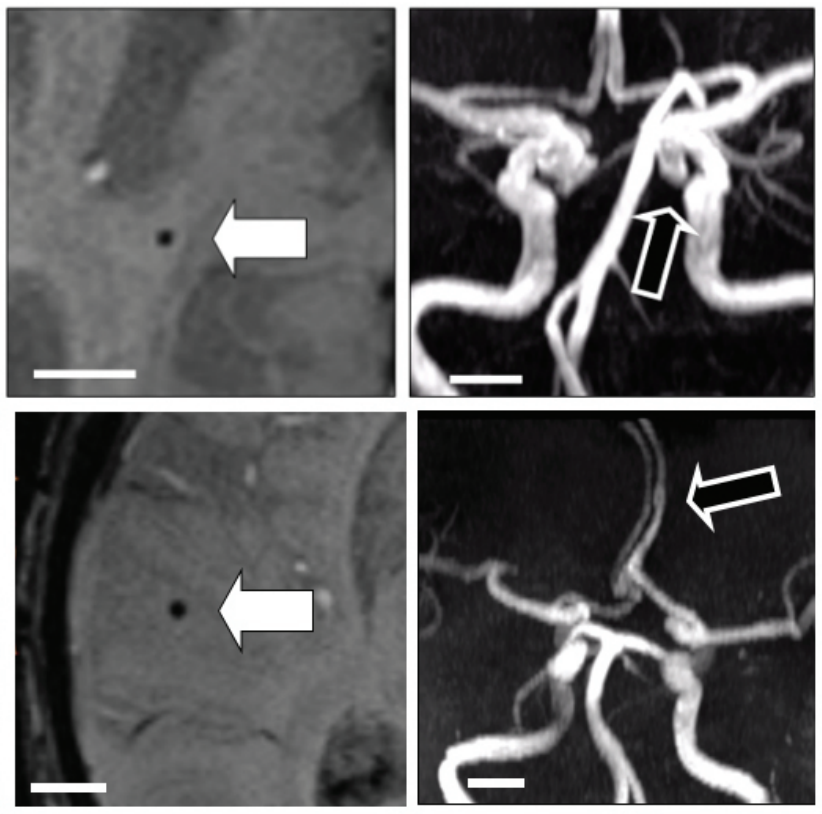

A
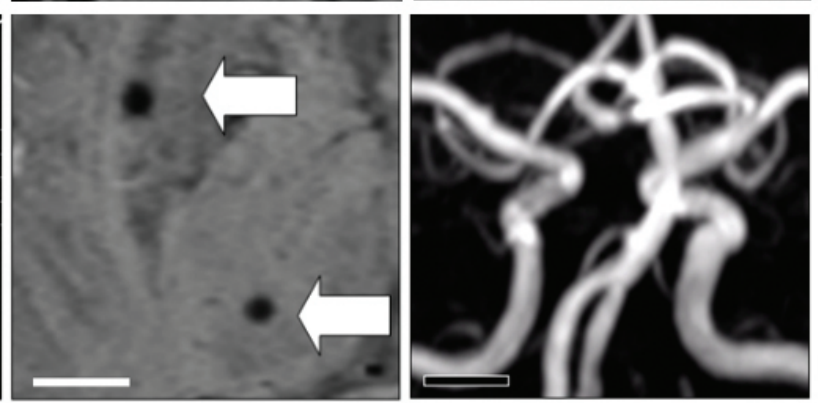

B

Figure 1. Patients with polycystic kidney disease (PKD) and having cerebral microbleeds (CMBs). (A) A 70-year-old woman had PKD with multiple cysts in bilateral kidney and liver (asterisks) noted by abdominal computed tomography (CT). The susceptibility-weighted imaging (SWI) shows a CMB (white arrow) in the left temporal area. The time-of-flight (TOF) image shows an intracranial aneurysm (black arrow) in the left distal internal carotid artery. (B) A 50-year-old man had PKD and the SWI shows a CMB (white arrow) in the right temporal area. The TOF image shows a fusiform aneurysm (black arrow) in the left anterior cerebral artery. (C) A 55-year-old woman had PKD and the SWI shows two CMBs (white arrows) in the right basal ganglion and thalamus. The TOF image does not show an aneurysm. Scale bar, $1 \mathrm{~cm}$.

Table 1. Determinants of the presence of cerebral microbleeds using logistic regression analysis

\begin{tabular}{|c|c|c|c|c|c|c|}
\hline \multirow{2}{*}{ Variable } & \multicolumn{3}{|c|}{ Univariable analysis } & \multicolumn{3}{|c|}{ Multivariable analysis } \\
\hline & $\mathrm{OR}$ & $P$ & $95 \% \mathrm{Cl}$ & Adjusted OR & $P$ & $95 \% \mathrm{Cl}$ \\
\hline Age (yr) & 1.07 & 0.008 & $1.02-1.13$ & 1.09 & 0.012 & $1.02-1.17$ \\
\hline Male sex & 1.54 & 0.412 & $0.55-4.35$ & & & \\
\hline Polycystic kidney disease & 5.25 & 0.013 & $1.41-19.5$ & 7.28 & 0.033 & $1.18-45.0$ \\
\hline Hypertension & 3.02 & 0.069 & $0.92-9.97$ & 1.04 & 0.962 & $0.25-4.31$ \\
\hline eGFR (mL/min/1.73 m²) & 0.98 & 0.002 & $0.96-0.99$ & 0.997 & 0.739 & $0.98-1.02$ \\
\hline
\end{tabular}

The model for multivariate analysis includes age, polycystic kidney disease, hypertension, and eGFR.

$\mathrm{OR}$, odds ratio; $\mathrm{Cl}$, confidence interval; eGFR, estimated glomerular filtration rate.

nants and eGFR level was a negative determinant for the detection of CMBs (Table 1). Using a multivariable logistic regression model, only older age (adjusted odds ratio [aOR], 1.09; $95 \%$ confidence interval $[\mathrm{Cl}], 1.02$ to 1.17 ) and the diagnosis of PKD (aOR, 7.28; 95\% Cl, 1.18 to 45.0$)$ remained independent determinants for the presence of CMBs.

In 49 patients receiving genetic testing, 22 (44.9\%) and 18 patients (36.7\%) were found to have PKD1 and PKD2 gene 
Missense: $50 \%$

Stopgain: $13.6 \%$

Insertion/deletion: $22.7 \%$

Splicing variant: $13.6 \%$

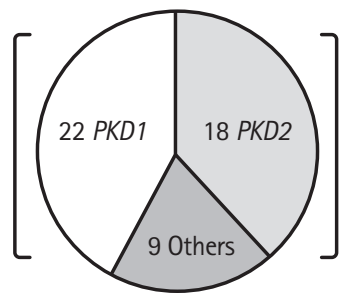

C2407T stopgain: $88.9 \%$

C681A stopgain: $5.6 \%$

Missense: $5.6 \%$
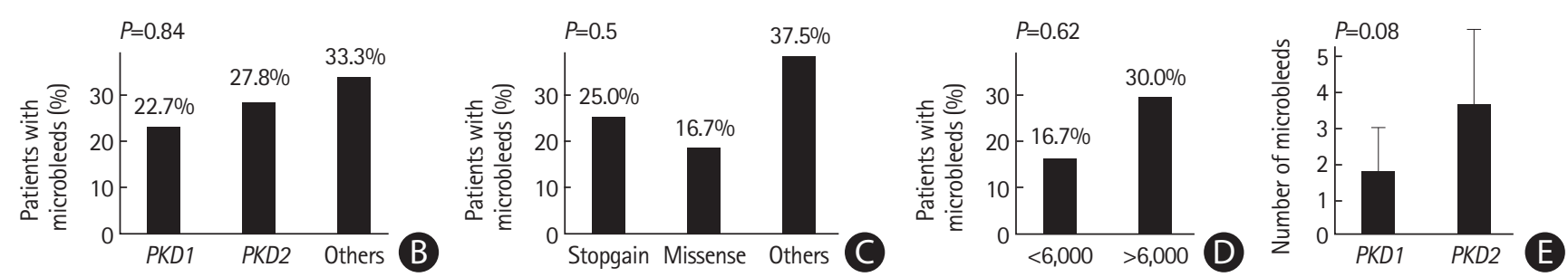

Figure 2. Genotype-phenotype correlation in 49 patients with polycystic kidney disease (PKD). (A) Twenty-two (44.9\%) and 18 (36.7\%) patients were found to have $P K D 1$ and $P K D 2$ gene causative variants, respectively. $(\mathrm{B}, \mathrm{C})$ The risk of having cerebral microbleeds (CMBs) did not differ significantly by the presence of different causative genes $(P=0.84)$ or variant types $(P=0.5)$. (D) For $P K D 1$ causative variants, no specific gene region $\left(5^{\prime}: 1-6,000\right.$ vs. $\left.3^{\prime}: 6,000-12,000\right)$ was associated with $C M B s(P=0.62)$. (E) Among PKD patients who had CMBs and received a genetic study $(n=13)$, patients with the $P K D 1$ causative variant showed a trend to have a higher average number of CMBs than those with PKD2 causative variants ( $3.8 \pm 2.0$ vs. $1.4 \pm 0.5, P=0.08)$. The total number of PKD patients with $\mathrm{CMBs}$ was relatively small $(n=15)$, impeding a complete phenotype and genotype analysis.

causative variants, respectively (Figure 2). No significant genotype correlated with CMBs in PKD patients. In 22 PKD patients who received the second MRI study with an average follow-up period of $36.5 \pm 12.4$ months, seven (31.8\%) developed new CMBs. For 12 PKD patients with no CMBs noted on the first MRI study, only one (8.3\%) had new CMBs on the follow-up MRI study; however, six of 10 PKD patients (60\%) with CMBs on the initial MRI developed new CMBs $(P=0.02)$. Patients with new $\mathrm{CMBs}$ during follow-up were older than those without new CMBs $(60.9 \pm 8.4$ years vs. $50.2 \pm 8.9$ years, $P=0.024)$.

The prevalence of CMBs in our PKD patients was about onefourth (27.3\%), much higher than that in the normal population of this study (6.7\%). Multivariable analysis revealed that older age and PKD diagnosis were the independent determinants for the presence of CMBs. During long-term follow-up, about one-third (31.8\%) of PKD patients also developed new CMBs. The present study demonstrates a novel vascular phenotype of PKD, i.e., CMBs, which indicates the relative structural fragility of cerebral small vessels in those with PKD.

Patients with cerebral small vessel disease (SVD) have been reported to more likely have CMBs. The present study is the first to demonstrate that those with PKD are prone to have CMBs. Other MRI markers found commonly in SVD, including an increase of white matter hyperintensity, the presence of lacune, and a high degree of enlarged perivascular space, ${ }^{3}$ were not features in PKD patients. The cerebral small vessel fragility in PKD might be the underlying pathophysiology that results in only CMB without any other parenchymal injury.
The distribution of CMBs in PKD patients were heterogeneous and found exclusively at lobar locations, exclusively deep and mixed regions in 46.7\%,33\% and 20\% of patients, respectively. In seven patients with exclusively lobar CMBs, three were younger than 55 years old and did not fulfill the Boston diagnostic criteria for cerebral amyloid angiopathy (CAA). ${ }^{13}$ Therefore, CAA is less likely in these relatively young patients when coexisting CAA is possible. Although chronic hypertension may be a risk factor for CMB formation in PKD patients, the fact that more than half of the CMBs were located in the lobar area suggests that factors other than hypertension may cause CMB formation. In addition, neither the presence of hypertension nor the eGFR level was significantly associated with CMBs in the multivariable model.

In summary, we report a novel phenotype of CMBs in patients with PKD that indicates the fragility of cerebral small vessels involving both deep penetrating and cortical-subcortical regions. The prevalence of CMBs is about one-fourth of PKD patients. Older age and the diagnosis of PKD are the only independent determinants of the occurrence of CMBs. Brain MRI studies in patients with PKD should emphasize the presence of not only intracranial aneurysm, but also CMBs.

\section{Supplementary materials}

Supplementary materials related to this article can be found online at https://doi.org/10.5853/jos.2019.02838. 


\section{References}

1. Cornec-Le Gall E, Alam A, Perrone RD. Autosomal dominant polycystic kidney disease. Lancet 2019;393:919-935.

2. Xu HW, Yu SO, Mei CL, Li MH. Screening for intracranial aneurysm in 355 patients with autosomal-dominant polycystic kidney disease. Stroke 2011;42:204-206.

3. Wardlaw JM, Smith EE, Biessels GJ, Cordonnier C, Fazekas F,

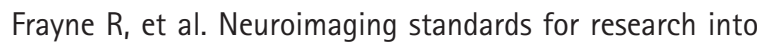
small vessel disease and its contribution to ageing and neurodegeneration. Lancet Neurol 2013;12:822-838.

4. Whelton PK, Carey RM, Aronow WS, Casey DE Jr, Collins KJ Dennison Himmelfarb C, et al. 2017 ACC/AHA/AAPA/ABC/ ACPM/AGS/APhA/ASH/ASPC/NMA/PCNA guideline for the prevention, detection, evaluation, and management of high blood pressure in adults: executive summary: a report of the American College of Cardiology/American Heart Association task force on clinical practice guidelines. Hypertension 2018;71:1269-1324.

5. Levey AS, Greene T, Kusek JW, Beck GJ. A simplified equation to predict glomerular filtration rate from serum creatinine. $J$ Am Soc Nephrol 2000;11:155A.

6. Tsai HH, Pasi M, Tsai LK, Chen YF, Lee BC, Tang SC, et al. Microangiopathy underlying mixed-location intracerebral hemorrhages/microbleeds: a PiB-PET study. Neurology2019;92:e774-e781.

7. Tsai HH, Tsai LK, Chen YF, Tang SC, Lee BC, Yen RF, et al. Correlation of cerebral microbleed distribution to amyloid burden in patients with primary intracerebral hemorrhage. Sci Rep 2017;7:44715.

8. Fazekas F, Chawluk JB, Alavi A, Hurtig HI, Zimmerman RA MR signal abnormalities at $1.5 \mathrm{~T}$ in Alzheimer's dementia and normal aging. AJR Am J Roentgenol 1987;149:351-356.
9. Pasi M, Boulouis G, Fotiadis P, Auriel E, Charidimou A, Haley K, et al. Distribution of lacunes in cerebral amyloid angiopathy and hypertensive small vessel disease. Neurology 2017;88: 2162-2168.

10. Charidimou A, Boulouis G, Pasi M, Auriel E, van Etten ES, Ha-

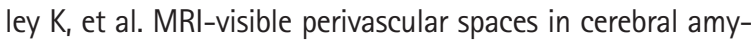
loid angiopathy and hypertensive arteriopathy. Neurology 2017;88:1157-1164.

11. Wu CC, Lin YH, Liu TC, Lin KN, Yang WS, Hsu CJ, et al. Identifying children with poor cochlear implantation outcomes using massively parallel sequencing. Medicine (Baltimore) 2015;94: e1073.

12. Rossetti S, Chauveau D, Kubly V, Slezak JM, Saggar-Malik $A K$, Pei $Y$, et al. Association of mutation position in polycystic kidney disease 1 (PKD1) gene and development of a vascular phenotype. Lancet 2003;361:2196-2201.

13. Greenberg SM, Charidimou A. Diagnosis of cerebral amyloid angiopathy: evolution of the Boston criteria. Stroke 2018:49:491497.

Correspondence: Juliana Tze-Wah Kao

Department of Internal Medicine, FuJen Catholic University Hospital, Fu Jen Catholic University, No. 69, Guizi Road, Taishan District, New Taipei City 24352, Taiwan

Tel: +886-2-29053957

Fax: +886-2-29896955

E-mail: twkao2@ntuh.gov.tw

Received: October 22, 2019

Revised: November 15, 2019

Accepted: January 14, 2020

This work was supported by grants from the National Taiwan University Hospital (108-S4110, 108-A147, and 201801072RSA-2-M04059).

The authors have no financial conflicts of interest. 


\section{Supplementary methods}

\section{Patients}

From November 2013 to September 2015, 55 polycystic kidney disease (PKD) patients without past history of stroke at the National Taiwan University Hospital were prospectively enrolled for brain magnetic resonance imaging (MRI) with susceptibility-weighted imaging (SWI). All patients received either abdominal computed tomography or MRI to confirm the diagnosis of PKD. Criteria for the diagnosis included at least two unilateral or bilateral cysts in persons younger than 30 years of age, at least two cysts in each kidney in persons 30 to 59 years of age and at least four cysts in each kidney in persons 60 years of age or older.' Demographic data, comorbidities, renal condition, blood platelet count, serum level of alanine aminotransferase, the usage of anti-platelet agent, and family history of each patient were comprehensively reviewed and recorded. The diagnosis of hypertension was defined as either treatment with antihypertensive drugs; or three home measurement of either a mean systolic blood pressure $\geq 130 \mathrm{~mm} \mathrm{Hg}$ or a mean diastolic blood pressure $\geq 80 \mathrm{~mm} \mathrm{Hg} .{ }^{4}$ The diagnosis of dyslipidemia was defined as either treatment with lipid lowering agents; or either serum total cholesterol level $>200 \mathrm{mg} / \mathrm{dL}$ or low density lipoprotein $>130 \mathrm{mg} / \mathrm{dL}$. We calculated the estimated glomerular filtration rate (eGFR) using the simplified Modification of Diet in Renal Disease equation as follows: eGFR $\left(\mathrm{mL} / \mathrm{min} / 1.73 \mathrm{~m}^{2}\right)=186 \times$ serum creatinine $(\mathrm{Scr})^{-1.154} \times$ Age $^{-0.203} \times 0.742$ (if female). ${ }^{5}$ This study was approved by the hospital Research Ethics Committee (201405049RIND and 201502049RINB). All patients provided written informed consent to participate.

Forty-five patients who visited the neurology outpatient clinics of National Taiwan University Hospital from July 2015 to March 2019 were chosen as control subjects. These patients had no prior stroke or family history of PKD. All received a brain MRI study and renal function test. Demographic data and comorbidities were recorded. The final diagnosis of these control subjects were primary headache (55.6\%), non-specific dizziness (20.0\%), anxiety (15.6\%), transient global amnesia (4.4\%), trigeminal neuralgia (2.2\%), and idiopathic hemifacial spasm $(2.2 \%)$. Only one patient had definite chronic kidney disease (eGFR=32 $\mathrm{mL} / \mathrm{min} / 1.73 \mathrm{~m}^{2}$ ) and his abdominal echo showed no evidence of polycystic kidneys.

\section{Brain magnetic resonance imaging}

All patients received a non-contrast brain MRI on a 3-Tesla MR scanner (Verio, TIM or mMR, Siemens, Erlangen, Germany). The imaging protocol consisted of axial T1-weighted, T2-weighted,
T2-fluid attenuated inversion recovery (FLAIR) images, SWI (covering the whole brain) and time-of-flight magnetic resonance angiography (for the intracranial vessels) sequences. Twenty-two PKD patients (40\%) received another follow-up brain MRI study over a period ranging from 14 to 60 months.

We obtained the SWI using a T2*-weighted gradient echo sequence with flip angle $15^{\circ}, T R / T E=28 / 20$ msec, matrix number $=221 \times 320, F O V=23 \mathrm{~cm}$, and slice thickness $=1.6 \mathrm{~mm}$. SWI and minimum intensity projection images, acquired by in-line post-processing of the magnitude and phase images, were used to evaluate the imaging findings in this study. Cerebral microbleeds (CMBs) were defined as small, round or ovoid hypointensities with a diameter less than $1 \mathrm{~cm}$ on SWI. ${ }^{3}$ We further categorized the location of CMBs into the lobar (cortical or corticalsubcortical areas in the fronto-parieto-temporo-occipital lobes) and deep regions (basal ganglion, thalamus and infratentorial areas). ${ }^{6}$ The intraobserver and interobserver reliability (к) was 0.84 and 0.70 in the determination of lobar CMB count and 0.76 and 0.72 in the determination of deep CMB count.?

Other neuroimaging markers of small vessel disease were rated according to the STandards for ReportIng Vascular changes on nEuroimaging (STRIVE) criteria [3], and evaluated as described previously. ${ }^{6}$ Briefly, white matter hyperintense (WMH) lesions were evaluated on FLAIR images using Fazekas scale. The WMH from moderate to severe was defined as Fazekas scale $\geq 2 .{ }^{6,8}$ Lacunes were defined as "round or ovoid, subcortical, fluid-filled (similar signal as cerebrospinal fluid) cavity, ranging 3 to $15 \mathrm{~mm}$ in diameter" ${ }^{6,9}$ and were evaluated in the supratentorial region, pons and cerebellum, respectively. Enlarged perivascular spaces (EPVSs) were defined as sharply delineated structures on T2-weighted imaging, measuring $<3 \mathrm{~mm}$ in diameter followed by the course of perforating or medullary vessels. The number of EPVS (on the side of the brain with more severe involvement) was counted in basal ganglion and centrum semiovale. We pre-specified a dichotomized classification of EPVS degree as high (score $>20$ ) or low (score $\leq 20$ ). ${ }^{6,10}$

\section{Genotyping with next-generation sequencing}

A total of 49 patients with PKD underwent the genetic test using next-generation sequencing (NGS) to screen the entire $P K D 1$ and $P K D 2$ genes for mutations while six patients refused to take the test. Briefly, blood samples were collected and genomic DNA (gDNA) was extracted using a Gentra Puregene Blood Kit (QIAGEN, Frederick, MD, USA) according to the manufacturer's protocol. The gDNA was fragmented to about 800 base pairs using Covaris (Covaris Inc., Woburn, MA, USA). The base pairs were then measured by Agilent Bioanalyzer 2100 (Agilent Technologies Inc., Santa Clara, CA, USA). Target enrich- 
ment was achieved using a customized NimbleGen SeqCap EZ Choice Kit (Roche NimbleGen Inc., Madison, WI, USA). NGS experiments were performed using Illumnian MiSeq at the Laboratory of Molecular Genetic Diagnostics, National Taiwan University Hospital (http://www.genetics-core-ntuh.tw/). The bioinformatics analysis pipeline was essentially the same as described in our previous work." All pathogenic variants detected through the NGS pipeline were further confirmed using the classical Sanger sequencing method. A genotype-phenotype correlation study reported that both PKD1 and PKD2 patients were at risk of intracranial aneurysm and the $5^{\prime}$ mutations were more commonly associated with intracranial aneurysm than 3 ' mutations in the PKD1 gene. ${ }^{12}$ Therefore, we also compared the percentage of patients having CMB between those with mutation at different $P K D 1$ gene regions (5':1-6,000 vs. $\left.3^{\prime}: 6,000-12,000\right)$. 
Supplementary Table 1. Characteristics of patients with and without polycystic kidney disease

\begin{tabular}{|c|c|c|c|}
\hline Characteristic & With PKD $(n=55)$ & Without PKD $(n=45)$ & $P$ \\
\hline Age (yr) & $51.3 \pm 10.9$ & $53.7 \pm 12.7$ & 0.324 \\
\hline Male sex & $21(38.2)$ & 15 (33.0) & 0.678 \\
\hline $\mathrm{eGFR}\left(\mathrm{mL} / \mathrm{min} / 1.73 \mathrm{~m}^{2}\right)$ & $58.2 \pm 38.0$ & $100.4 \pm 27.7$ & $<0.001$ \\
\hline Dialysis & $12(21.8)$ & $0(0)$ & $<0.001$ \\
\hline Hypertension & 43 (78.2) & $19(42.2)$ & $<0.001$ \\
\hline Diabetes & $3(5.5)$ & $1(2.2)$ & 0.625 \\
\hline Dyslipidemia & $9(16.4)$ & $6(13.0)$ & 0.781 \\
\hline Smoking & $4(7.3)$ & $3(6.7)$ & 1.000 \\
\hline Anti-platelet agent & $4(7.3)$ & $0(0)$ & 0.125 \\
\hline Cerebral microbleeds & 15 (27.3) & $3(6.7)$ & 0.009 \\
\hline Intracranial aneurysm & $6(10.9)$ & $0(0)$ & 0.031 \\
\hline Cavernous angioma & $5(9.1)$ & $1(2.2)$ & 0.219 \\
\hline Intraventricular cyst & $2(3.6)$ & $0(0)$ & 0.500 \\
\hline Moderate to severe white matter intensity* & 10 (18.2) & $3(6.7)$ & 0.135 \\
\hline Presence of lacune & $6(10.9)$ & $3(6.7)$ & 0.508 \\
\hline High degree of EPVS at $\mathrm{CSO}^{+}$ & $12(21.8)$ & $8(17.8)$ & 0.802 \\
\hline High degree of EPVS at $\mathrm{BG}^{+}$ & $7(12.7)$ & $3(6.7)$ & 0.505 \\
\hline
\end{tabular}

Values are presented as mean \pm standard deviation or number (\%).

PKD, polycystic kidney disease; eGFR, estimated glomerular filtration rate; EPVS, enlarged perivascular space; CSO, centrum semiovale; BG, basal ganglion.

${ }^{*}$ Moderate to severe white matter intensity is defined as Fazekas scale $\geq 2 ;{ }^{+}$High degree of EPVS is defined as score $>20$.

Supplementary Table 2. Characteristics of patients having polycystic kidney disease with and without cerebral microbleeds

\begin{tabular}{|c|c|c|c|}
\hline Characteristic & With CMBs $(n=15)$ & Without CMBs $(n=40)$ & $P$ \\
\hline Age (yr) & $58.4 \pm 10.8$ & $48.7 \pm 9.8$ & 0.002 \\
\hline Male sex & $6(40)$ & $15(37.5)$ & 1.000 \\
\hline eGFR $\left(\mathrm{mL} / \mathrm{min} / 1.73 \mathrm{~m}^{2}\right)$ & $45.1 \pm 39.9$ & $63.1 \pm 36.5$ & 0.120 \\
\hline Dialysis & $6(40.0)$ & $6(15.0)$ & 0.068 \\
\hline Hypertension & 14 (93.3) & $29(72.5)$ & 0.147 \\
\hline Diabetes & $0(0)$ & $3(7.5)$ & 0.554 \\
\hline Dyslipidemia & $4(26.7)$ & $5(12.5)$ & 0.236 \\
\hline Smoking & $2(13.3)$ & $2(5.0)$ & 0.298 \\
\hline Alcohol consumption & $2(13.3)$ & $2(5.0)$ & 0.298 \\
\hline Anti-platelet agent & $1(6.7)$ & $3(7.5)$ & 1.000 \\
\hline Family history of ICH & $0(0)$ & $3(7.5)$ & 0.554 \\
\hline Alanine Aminotransferase (U/L) & $15.1 \pm 5.8$ & $14.8 \pm 7.9$ & 0.906 \\
\hline Platelet count $(\mathrm{k} / \mu \mathrm{L})$ & $178 \pm 63$ & $207 \pm 72$ & 0.200 \\
\hline Intracranial aneurysm & $2(13.3)$ & $4(10)$ & 0.660 \\
\hline Moderate to severe white matter intensity* & $4(26.7)$ & $6(15.0)$ & 0.434 \\
\hline Presence of lacune & $1(6.7)$ & $5(12.5)$ & 1.000 \\
\hline High degree of EPVS at $\mathrm{CSO}^{+}$ & $5(33.3)$ & $7(17.5)$ & 0.274 \\
\hline High degree of EPVS at $\mathrm{BG}^{+}$ & $2(13.3)$ & $5(12.5)$ & 1.000 \\
\hline
\end{tabular}

Values are presented as mean \pm standard deviation or number $(\%)$.

$\mathrm{CMB}$, cerebral microbleed; $\mathrm{CSO}$, centrum semiovale; eGFR, estimated glomerular filtration rate; ICH, intracranial hemorrhage; EPVS, enlarged perivascular space; $\mathrm{CSO}$, centrum semiovale; $\mathrm{BG}$, basal ganglion.

${ }^{*}$ Moderate to severe white matter intensity is defined as Fazekas scale $\geq 2 ;{ }^{+}$High degree of EPVS is defined as score $>20$. 